\title{
Effects of the van der Waals Force on the Dynamics Performance for a Micro Resonant Pressure Sensor
}

\author{
Lizhong Xu, Yulei Liu, and Xiaorui Fu \\ Mechanical Engineering Institute, Yanshan University, Qinhuangdao 066004, China \\ Correspondence should be addressed to Lizhong Xu; xlz@ysu.edu.cn
}

Received 12 June 2015; Revised 4 November 2015; Accepted 16 November 2015

Academic Editor: Evgeny Petrov

Copyright (C) 2016 Lizhong Xu et al. This is an open access article distributed under the Creative Commons Attribution License, which permits unrestricted use, distribution, and reproduction in any medium, provided the original work is properly cited.

\begin{abstract}
The micro resonant pressure sensor outputs the frequency signals where the distortion does not take place in a long distance transmission. As the dimensions of the sensor decrease, the effects of the van der Waals forces should be considered. Here, a coupled dynamic model of the micro resonant pressure sensor is proposed and its coupled dynamic equation is given in which the van der Waals force is considered. By the equation, the effects of the van der Waals force on the natural frequencies and vibration amplitudes of the micro resonant pressure sensor are investigated. Results show that the natural frequency and the vibrating amplitudes of the micro resonant pressure sensor are affected significantly by van der Waals force for a small clearance between the film and the base plate, a small initial tension stress of the film, and some other conditions.
\end{abstract}

\section{Introduction}

Microelectromechanical Systems (MEMS) have advantages such as compact structure, low cost, small power loss, high response speed, and high accuracy $[1,2]$. The micro resonant sensor is more attractive because it outputs the frequency signals where distortion error does not occur and it is suitable for the distant range transmission.

In 1990-1991, the micro resonant pressure sensors with heat excitation and electromagnetic excitation were developed $[3,4]$. In this year, a micro resonant pressure sensor with electrostatic excitation was proposed which can achieve an accuracy of $100 \mathrm{ppm}$ at the temperature range of the automobile operation [5]. A new alloy based on transition metals was developed to obtain large amplitude of the resonant longitudinal magnetoelastic waves which can be used to improve performance of the micro resonant sensor [6]. A type of NEMS double Si3N4 resonant beams pressure sensor was presented and the sensitivity of the sensor is getting to $498.24 \mathrm{~Hz} / \mathrm{kPa}$ [7]. An electrothermally excited dual beams silicon resonant pressure sensor with temperature compensation was proposed and the experimental results indicate that the maxim residual error is $1.8 \mathrm{kPa}$ in the working temperature range from -40 to $60^{\circ} \mathrm{C}$ [8]. The nonlinear dynamics of a resonant silicon bridge pressure sensor with electrothermal excitation was investigated in which the measured pressure, the heating effect of the electrothermal excitation, and the residual internal force in the bridge were considered [9]. The sensitivity of the micro resonant pressure sensor with docks was investigated [10]. A novel resonant pressure sensor with an improved micromechanical doubleended tuning fork resonator packaged in dry air at atmospheric pressure was presented in which the fundamental frequency of the resonant pressure sensor is approximately $34.55 \mathrm{kHz}$ with a pressure sensitivity of $20.77 \mathrm{~Hz} / \mathrm{kPa}$ [11]. A new stress isolation method based resonant pressure sensor was presented to minimize thermal stresses arising from device packaging due to thermal mismatches between the silicon sensor body and its housing materials [12].

In a word, a number of studies about micro resonant pressure sensor have been done. However, in a micro resonant pressure sensor, as the clearance between the resonant film and basement is small enough, the effects of the van der Waals force will become obvious.

The influence of surface effects on the pull-in instability of a cantilever nanoactuator was investigated incorporating the influence of the Casimir attraction and van der Waals force $[13,14]$. Besides it, the effects of the molecular forces on the free vibration of electromechanical integrated electrostatic harmonic actuator were studied as well [15]. 


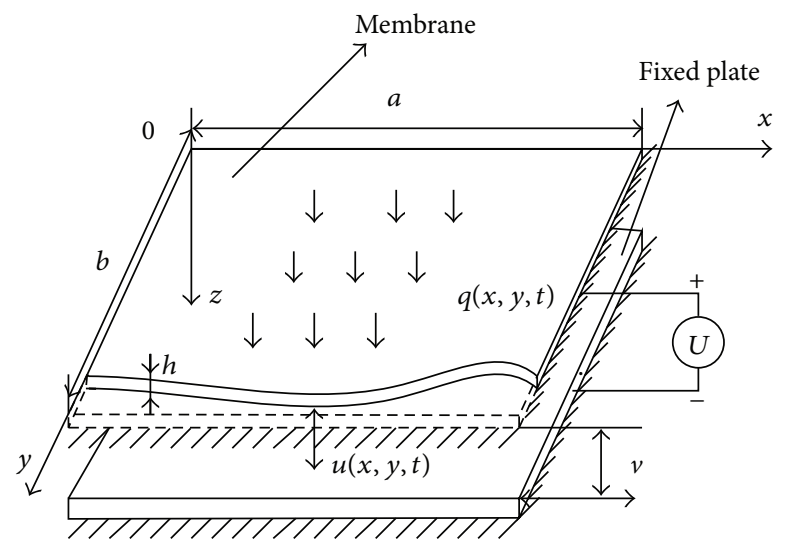

FIGURE 1: Multifields coupled dynamic model of a micro film.

Above-mentioned studies mainly focus on dynamics performance of the micro ring (see [15]) and micro beam (other references) which is only controlled by one partial differential equation. In our micro resonant pressure sensor, the resonator is the micro film in which the dynamics performance is controlled by two partial differential equations. So, the effect problem of the van der Waals force on the dynamics performance of a micro resonant pressure sensor is more complicated and has not been resolved yet.

In this paper, a coupled dynamic model of the micro resonant pressure sensor is proposed and its coupled dynamic equation is given in which the van der Waals force is considered. Using these equations, the effects of the van der Waals force on the natural frequencies and vibration amplitudes of the micro resonant pressure sensor are investigated. Results show that the natural frequency and the vibrating amplitudes of the micro resonant pressure sensor are affected significantly by van der Waals force for a small clearance between the film and the base plate, a small initial tension stress of the film, and some other conditions. These results can be used to improve design about dynamics performance for the micro resonant pressure sensor.

\section{Vibration Equations}

Figure 1 illustrates a micro film in the micro resonant pressure sensor. The electrostatic force and the van der Waals force are applied to the micro film. Its boundary condition is that two sides are fixed and two sides are free. Here, the dynamics partial differential equation of the film under uniform tension is not applicable. Two dynamics partial differential equations of the orthotropic film can be used. The vibration equations of the orthotropic micro film are [16]

$$
\begin{aligned}
& \rho h \frac{\partial^{2} w}{\partial t^{2}}-\left(\sigma_{0 x}+\frac{\partial^{2} \varphi}{\partial y^{2}}\right) h \frac{\partial^{2} w}{\partial x^{2}}-\left(\sigma_{y 0}+\frac{\partial^{2} \varphi}{\partial x^{2}}\right) h \frac{\partial^{2} w}{\partial y^{2}} \\
& =q \\
& \frac{1}{E_{1}} \frac{\partial^{4} \varphi}{\partial y^{4}}+\frac{1}{E_{2}} \frac{\partial^{4} \varphi}{\partial x^{4}}=\left(\frac{\partial^{2} w}{\partial x \partial y}\right)^{2}-\frac{\partial^{2} w}{\partial x^{2}} \frac{\partial^{2} w}{\partial y^{2}}
\end{aligned}
$$

where $\rho$ is the mass density of the film, $t$ is the time, $v$ is the initial clearance between the micro film and base plate, $U$ is the voltage between the micro film and base plate, $w$ is the transverse displacement of the film, $h$ is the thickness of the film, $\varphi$ is the stress function, $\sigma_{0 x}$ is the initial tension stress in $x$ direction of the film, $\sigma_{y 0}$ is the initial tension stress in $y$ direction of the film, $x$ is the coordinate in the film length direction, $y$ is the coordinate in the film width direction, $E$ is the modulus of elasticity of the micro film material, and $q(x, y, t)$ is the transverse load per unit area on the film.

The electrostatic force per unit area is

$$
q_{e}=\frac{F_{e}}{A}=\frac{\varepsilon_{0} \varepsilon_{r}}{2}\left(\frac{U}{v-w}\right)^{2} \text {, }
$$

where $F_{e}$ is the electrostatic force applied to the micro film, $A$ is the area of the micro film, $\varepsilon_{0}$ is permittivity constant of free space, and $\varepsilon_{r}$ is relative dielectric constant of the insulating layer.

The van der Waals force per unit area between the film and fixed plate is

$$
q_{r 3}=\frac{A}{6 \pi(v-w)^{3}},
$$

where $A$ is the Hamaker constant: $A=10^{-19} \mathrm{~J}$.

The damping force per unit area from air is

$$
q_{c}=c_{a} \frac{\partial w}{\partial t}
$$

Here, $c_{a}$ is the damping coefficient of the gas.

Thus, the total force per unit area on the film is

$$
q(x, y, t)=q_{e}+q_{r 3}+q_{c}
$$

The displacement $w$ of the micro film consists of a static component $w_{s}$ and a dynamic one $\Delta w$ :

$$
w=w_{s}+\Delta w
$$


The load $q(x, y, t)$ of static $\left(q_{s}\right)$ and dynamic $(\Delta q)$ components is

$$
\begin{aligned}
q(x, y, t) & =q_{s}+\Delta q \\
q_{s} & =\frac{U_{0}^{2} \varepsilon_{0} \varepsilon_{r}}{2\left(v-w_{s}\right)^{2}}+\frac{A}{6 \pi\left(v-w_{s}\right)^{3}} .
\end{aligned}
$$

From $\Delta q=(d q / d w) \Delta w$, we know that

$$
\Delta q=\frac{U_{0}^{2} \varepsilon_{0} \varepsilon_{r}}{\left(v-w_{s}\right)^{3}} \Delta w+\frac{A}{2 \pi\left(v-w_{s}\right)^{4}} \Delta w-c_{a} \frac{\partial \Delta w}{\partial t},
$$

where $c_{a}=\mu b^{3} /\left(v-w_{s}\right)^{3}$ and $\mu$ is the gas viscosity: $\mu=1.86 \times$ $10^{-5} \mathrm{~N} \cdot \mathrm{S} \cdot \mathrm{m}^{-2}$.

Substituting (6) and (7) into (1a) and (1b) yields the following equations:

$$
\begin{gathered}
\left(\sigma_{0 x}+\frac{\partial^{2} \varphi}{\partial y^{2}}\right) h \frac{\partial^{2} w_{s}}{\partial x^{2}}-\left(\sigma_{y 0}+\frac{\partial^{2} \varphi}{\partial x^{2}}\right) h \frac{\partial^{2} w_{s}}{\partial y^{2}}=q_{s}, \\
\frac{1}{E_{1}} \frac{\partial^{4} \varphi}{\partial y^{4}}+\frac{1}{E_{2}} \frac{\partial^{4} \varphi}{\partial x^{4}}=\left(\frac{\partial^{2} w_{s}}{\partial x \partial y}\right)^{2}-\frac{\partial^{2} w_{s}}{\partial x^{2}} \frac{\partial^{2} w_{s}}{\partial y^{2}}, \\
\rho h \frac{\partial^{2} \Delta w}{\partial t^{2}}-\left(\sigma_{0 x}+\frac{\partial^{2} \Delta \varphi}{\partial y^{2}}\right) h \frac{\partial^{2} \Delta w}{\partial x^{2}} \\
-\left(\sigma_{y 0}+\frac{\partial^{2} \Delta \varphi}{\partial x^{2}}\right) h \frac{\partial^{2} \Delta w}{\partial y^{2}}=\Delta q, \\
\frac{1}{E_{1}} \frac{\partial^{4} \Delta \varphi}{\partial y^{4}}+\frac{1}{E_{2}} \frac{\partial^{4} \Delta \varphi}{\partial x^{4}}=\left(\frac{\partial^{2} \Delta w}{\partial x \partial y}\right)^{2}-\frac{\partial^{2} \Delta w}{\partial x^{2}} \frac{\partial^{2} \Delta w}{\partial y^{2}} .
\end{gathered}
$$

\section{Free Vibration}

For a micro pressure sensor, two ends of the film are fixed and other two ends of the film are free; the boundary conditions are

$$
\begin{gathered}
\Delta w(0, y, t)=\Delta w(a, y, t)=0, \\
\left.\frac{\partial \Delta w}{\partial y}\right|_{y=0}=\left.\frac{\partial \Delta w}{\partial y}\right|_{y=b}=0 .
\end{gathered}
$$

For the boundary conditions, the solutions of (11a) and (11b) can be given as

$$
\begin{aligned}
& \Delta w(x, y, t)=W(x, y) T(t), \\
& \Delta \varphi(x, y, t)=\phi(x, y) T^{2}(t) .
\end{aligned}
$$

Here,

$$
W(x, y)=A \sin \frac{m \pi x}{a} \cos \frac{n \pi y}{b} .
$$

Thus

$$
\Delta w(x, y, t)=A \sin \frac{m \pi x}{a} \cos \frac{n \pi y}{b} T(t) .
$$

Substituting (13b) and (13d) into (11b) yields

$$
\begin{aligned}
\frac{1}{E_{1}} & \frac{\partial^{4} \phi}{\partial y^{4}}+\frac{1}{E_{2}} \frac{\partial^{4} \phi}{\partial x^{4}} \\
& =\frac{m^{2} n^{2} \pi^{4}}{2 a^{2} b^{2}}\left(\cos \frac{2 m \pi x}{a}-\cos \frac{2 n \pi y}{b}\right) .
\end{aligned}
$$

Letting $\phi(x, y)=\alpha \cos (2 m \pi x / a)-\beta \cos (2 n \pi y / b)$, and substituting it into (14), yields

$$
\begin{aligned}
& \alpha=\frac{E_{2} n^{2} a^{2}}{32 m^{2} b^{2}}, \\
& \beta=\frac{E_{1} m^{2} b^{2}}{32 n^{2} a^{2}} .
\end{aligned}
$$

Substituting it into (13b) yields

$$
\begin{aligned}
& \Delta \varphi(x, y, t) \\
& =\left(\frac{E_{2} n^{2} a^{2}}{32 m^{2} b^{2}} \cos \frac{2 m \pi x}{a}-\frac{E_{1} m^{2} b^{2}}{32 n^{2} a^{2}} \cos \frac{2 n \pi y}{b}\right) T^{2}(t) .
\end{aligned}
$$

Letting $E=U_{0}^{2} \varepsilon_{0} \varepsilon /\left(v-w_{0}\right)^{3}+A / 2 \pi\left(v-w_{0}\right)^{4}$ (here, $w_{0}$ is the average static displacement of the micro film) and substituting (13b), (13d), and (9) into (11a) and using Galerkin method yield

$$
\begin{gathered}
\ddot{T}+\left[\frac{\pi^{2}}{\rho}\left(\frac{m^{2}}{a^{2}} \sigma_{0 x}+\frac{n^{2}}{b^{2}} \sigma_{y 0}\right)-\frac{E}{\rho h}\right] T+\frac{C_{a}}{\rho h} \dot{T} \\
+\frac{3 \pi^{4}}{16 \rho}\left(\frac{E_{1} m^{4}}{a^{4}}-\frac{E_{2} n^{4}}{b^{4}}\right) T^{3}=0 .
\end{gathered}
$$

Letting $\varepsilon=h^{2} / a b,(17)$ can be changed into the following form:

$$
\ddot{T}+\omega_{0}^{2} T=\varepsilon\left(\alpha_{1} T^{3}+\alpha_{2} \dot{T}\right),
$$

where $\omega_{0}^{2}=\left(\pi^{2} / \rho\right)\left(\left(m^{2} / a^{2}\right) \sigma_{0 x}+\left(n^{2} / b^{2}\right) \sigma_{y 0}\right)-E / \rho h, \alpha_{1}=$ $-\left(3 \pi^{4} a b / 16 \rho h\right)\left(E_{1} m^{4} / a^{4}-E_{2} n^{4} / b^{4}\right)$, and $\alpha_{2}=-a b C_{a} / \rho h^{2}$. 
Letting $f(T, \dot{T})=\left(\alpha_{1} T^{3}+\alpha_{2} \dot{T}\right)$ and $\psi=\omega_{0} t+\theta$, if $\varepsilon=0$, then (18) can be changed into the following form:

$$
\frac{d T}{d t}=-a \omega_{0} \sin \psi
$$

Letting $T=a \cos \psi$, here $a$ and $\psi$ are the function of the time. Thus

$$
\frac{d T}{d t}=\frac{d a}{d t} \cos \psi-a\left(\omega_{0}+\frac{d \theta}{d t}\right) \sin \psi
$$

From (19) and (20), we can give

$$
\begin{aligned}
& \frac{d a}{d t} \cos \psi-a \frac{d \theta}{d t} \sin \psi=0, \\
& \frac{d^{2} T}{d t^{2}}=-\frac{d a}{d t} \omega_{0} \sin \psi-a \omega_{0}\left(\omega_{0}+\frac{d \theta}{d t}\right) \cos \psi
\end{aligned}
$$

Substituting $T=a \cos \psi$ and (22) into (18) yields

$$
\begin{gathered}
-\frac{d a}{d t} \omega_{0} \sin \psi-a \omega_{0} \frac{d \theta}{d t} \cos \psi \\
=\varepsilon f\left(a \cos \psi,-a \omega_{0} \sin \psi\right) .
\end{gathered}
$$

From (21) and (23), we can give

$$
\begin{aligned}
& \frac{d a}{d t}=-\frac{\varepsilon}{\omega_{0}} f\left(a \cos \psi,-a \omega_{0} \sin \psi\right) \sin \psi \\
& \frac{d \theta}{d t}=-\frac{\varepsilon}{a \omega_{0}} f\left(a \cos \psi,-a \omega_{0} \sin \psi\right) \cos \psi
\end{aligned}
$$

The right parts of (24a) and (24b) can be written in Fourier series:

$$
\begin{aligned}
& f\left(a \cos \psi,-a \omega_{0} \sin \psi\right) \sin \psi \\
& =A_{0}(a)+\sum_{n=1}^{\infty}\left(A_{n} \cos n \psi+B_{n} \sin n \psi\right), \\
& f\left(a \cos \psi,-a \omega_{0} \sin \psi\right) \cos \psi \\
& =C_{0}(a)+\sum_{n=1}^{\infty}\left(C_{n} \cos n \psi+D_{n} \sin n \psi\right),
\end{aligned}
$$

TABle 1: Parameters of the micro film.

\begin{tabular}{lcccccc}
\hline $\begin{array}{l}a \\
(\mathrm{~mm})\end{array}$ & $\begin{array}{c}b \\
(\mathrm{~mm})\end{array}$ & $\begin{array}{c}h \\
(\mu \mathrm{m})\end{array}$ & $\begin{array}{c}\varepsilon_{0} \\
\left(\mathrm{C}^{2} \cdot \mathrm{N}^{-1} \cdot \mathrm{m}^{-2}\right)\end{array}$ & $\begin{array}{c}v \\
(\mu \mathrm{m})\end{array}$ & $\begin{array}{c}E \\
(\mathrm{GPa})\end{array}$ & $\begin{array}{c}\rho \\
\left(\mathrm{kg} / \mathrm{m}^{3}\right)\end{array}$ \\
\hline 2 & 1 & 5 & $8.85 \times 10^{-12}$ & 0.5 & 190 & 2330 \\
\hline
\end{tabular}

where

$$
\begin{aligned}
A_{0}(a) & =\frac{1}{2 \pi} \int_{0}^{2 \pi} \sin \psi f\left(a \cos \psi,-a \omega_{0} \sin \psi\right) d \psi \\
& =-\frac{1}{2} \alpha_{2} a \omega_{0}, \\
C_{0}(a) & =\frac{1}{2 \pi} \int_{0}^{2 \pi} \cos \psi f\left(a \cos \psi,-a \omega_{0} \sin \psi\right) d \psi \\
& =\frac{3}{8} \alpha_{1} a^{3} .
\end{aligned}
$$

In (25a) and (25b), only the average values $A_{0}(a)$ and $C_{0}(a)$ are kept. Substituting (25a) and (25b) into (24a) and (24b) yields

$$
\begin{aligned}
& \frac{d a}{d t}=-\frac{\varepsilon}{\omega_{0}} A_{0}(a)=\frac{1}{2} \varepsilon \alpha_{2} a, \\
& \frac{d \theta}{d t}=-\frac{\varepsilon}{a \omega_{0}} C_{0}(a)=-\frac{3}{8} \frac{\varepsilon \alpha_{1} a^{2}}{\omega_{0}}, \\
& \frac{d \psi}{d t}=\omega_{0}-\frac{\varepsilon}{a \omega_{0}} C_{0}(a)=\omega_{0}-\frac{3}{8} \frac{\varepsilon \alpha_{1} a^{2}}{\omega_{0}} .
\end{aligned}
$$

From (27a), (27b), and (27c), we know that

$$
\begin{aligned}
& a=a_{0} e^{\alpha_{2} \varepsilon t / 2}, \\
& \psi=\left(\omega_{0}-\frac{3 \alpha_{1} a^{2} \varepsilon}{8 \omega_{0}}\right) t+\psi_{0},
\end{aligned}
$$

where $a_{0}$ is the initial displacement and $\psi_{0}$ is the initial phase. Letting $\psi_{0}=0$, we obtain

$$
\begin{aligned}
T(t) & =a_{0} e^{\alpha_{2} \varepsilon t / 2} \cos \left[\left(\omega_{0}-\frac{3 \alpha_{1} a_{0}^{2} e^{\alpha_{2} \varepsilon t} \varepsilon}{8 \omega_{0}}\right) t\right], \\
\omega & =\omega_{0}-\frac{3 \alpha_{1} a_{0}^{2} e^{\alpha_{2} \varepsilon t} \varepsilon}{8 \omega_{0}} .
\end{aligned}
$$

Substituting (29a) and (29b) into (13d) yields

$$
\begin{gathered}
w(x, y, t)=\sum_{m=1}^{\infty} \sum_{n=1}^{\infty} \sin \frac{m \pi x}{a} \cos \frac{n \pi y}{b} \\
\cdot\left\{a_{0} e^{\alpha_{2} \varepsilon t / 2} \cos \left[\left(\omega_{0}-\frac{3 \alpha_{1} a_{0}^{2} e^{\alpha_{2} \varepsilon t} \varepsilon}{8 \omega_{0}}\right) t\right]\right\} .
\end{gathered}
$$

\section{Results and Discussions}

Above equations are utilized for the free vibration analysis of the micro resonant pressure sensor. The parameters of the numerical example are shown in Table 1 (here, 
TABLE 2: Natural frequency of the micro pressure sensor for various clearances ( $\mathrm{rad} / \mathrm{s})$.

\begin{tabular}{|c|c|c|c|c|c|c|c|}
\hline & $\omega$ & $\omega_{f}$ & $\eta(\%)$ & & $\omega$ & $\omega_{f}$ & $\eta(\%)$ \\
\hline \multicolumn{8}{|c|}{$v=5.0 \times 10^{-7} \mathrm{~m}$} \\
\hline Mode $(1,1)$ & 28163.51 & 24990.48 & 11.27 & Mode $(3,3)$ & 207730.60 & 207324.24 & 0.196 \\
\hline Mode $(2,2)$ & 129142.32 & 128487.67 & 0.507 & Mode $(4,4)$ & 283224.23 & 282926.33 & 0.105 \\
\hline \multicolumn{8}{|c|}{$v=5.5 \times 10^{-7} \mathrm{~m}$} \\
\hline Mode $(1,1)$ & 49598.20 & 48671.80 & 1.868 & Mode $(3,3)$ & 211704.51 & 211489.39 & 0.102 \\
\hline Mode $(2,2)$ & 135442.01 & 135105.52 & 0.248 & Mode $(4,4)$ & 286151.65 & 285992.53 & 0.056 \\
\hline \multicolumn{8}{|c|}{$v=6.0 \times 10^{-7} \mathrm{~m}$} \\
\hline Mode $(1,1)$ & 58272.73 & 57813.02 & 0.789 & Mode $(3,3)$ & 213903.08 & 213728.30 & 0.082 \\
\hline Mode $(2,2)$ & 138853.40 & 138661.11 & 0.138 & Mode $(4,4)$ & 287782.02 & 287689.29 & 0.032 \\
\hline
\end{tabular}

TABLE 3: Natural frequency of the micro pressure sensor for various initial tension stresses (rad/s).

\begin{tabular}{|c|c|c|c|c|c|c|c|}
\hline & $\omega$ & $\omega_{f}$ & $\eta(\%)$ & & $\omega$ & $\omega_{f}$ & $\eta(\%)$ \\
\hline \multicolumn{8}{|c|}{$\sigma_{0 x}=4.5 \times 10^{3} \mathrm{KN} / \mathrm{m}$} \\
\hline Mode $(1,1)$ & 16238.77 & 9748.79 & 39.966 & Mode $(3,3)$ & 195925.07 & 195494.18 & 0.220 \\
\hline Mode $(2,2)$ & 120663.99 & 119963.07 & 0.581 & Mode $(4,4)$ & 267851.08 & 267536.05 & 0.118 \\
\hline \multicolumn{8}{|c|}{$\sigma_{0 x}=5.0 \times 10^{3} \mathrm{KN} / \mathrm{m}$} \\
\hline Mode $(1,1)$ & 28163.51 & 24990.48 & 11.27 & Mode $(3,3)$ & 207730.60 & 207324.24 & 0.196 \\
\hline Mode $(2,2)$ & 129142.32 & 128487.67 & 0.507 & Mode $(4,4)$ & 283224.23 & 282926.33 & 0.105 \\
\hline \multicolumn{8}{|c|}{$\sigma_{0 x}=5.5 \times 10^{3} \mathrm{KN} / \mathrm{m}$} \\
\hline Mode $(1,1)$ & 36368.50 & 33970.71 & 6.594 & Mode $(3,3)$ & 218900.35 & 218514.77 & 0.176 \\
\hline Mode $(2,2)$ & 137097.33 & 136480.84 & 0.450 & Mode $(4,4)$ & 297804.85 & 297521.54 & 0.095 \\
\hline
\end{tabular}

$a_{0}=2 \times 10^{-7} \mathrm{~m}, U_{0}=0.4 \mathrm{~V}$, and $\left.\sigma_{y 0}=0\right)$. Natural frequencies of the micro resonant pressure sensor under various clearances are shown in Table 2 (here, $\sigma_{0 x}=5.0 \times 10^{3} \mathrm{KN} / \mathrm{m}$ ). Natural frequencies of the micro resonant pressure sensor under various initial tension stresses are shown in Table 3 (here, $v=5.0 \times 10^{-7} \mathrm{~m}$ ). $\omega$ is the natural frequency of the sensor without considering the van der Waals force, $\omega_{f}$ is the natural frequency of the sensor with considering the van der Waals force, and $\eta$ is the relative error between them. From Tables 2 and 3, the following observations are worth noting.

(1) Without considering the van der Waals force, the natural frequencies of the micro sensor are larger than those in the case of considering the van der Waals force. It is because the van der Waals force system is equivalent to a soft spring system. The van der Waals force can cause decrease of the natural frequencies of the micro sensor.

(2) The deviation between the natural frequencies of the micro sensor with and without the van der Waals force decreases with increasing the order number of the mode. For mode $(1,1)$, the relative error between the natural frequencies is $11.27 \%\left(v=5.0 \times 10^{-7} \mathrm{~m}\right)$. For mode $(4,4)$, the relative error between the natural frequencies is $0.1 \%\left(v=5.0 \times 10^{-7} \mathrm{~m}\right)$. It shows that influence of the van der Waals force on the natural frequencies decreases with increasing the order number of the mode.

(3) At an initial tension stress, the natural frequency of the sensor increases significantly with increasing the clearance between the film and the base plate.

If the clearance grows, the deviation between the natural frequencies of the sensor with and without the van der Waals force drops. At $v=5.0 \times 10^{-7} \mathrm{~m}$, the relative error between the natural frequencies with and without the van der Waals force is $11.27 \%$ for mode $(1,1)$. At $v=6.0 \times 10^{-7} \mathrm{~m}$, the relative error between the natural frequencies with and without the van der Waals force is $0.79 \%$.

(4) At a constant clearance between the film and the base plate, the natural frequency of the sensor increases significantly with increasing the film tension. As the film tension grows, the deviation between the natural frequencies of the sensor with and without the van der Waals force decreases. At $\sigma_{0 x}=4.5 \times 10^{3} \mathrm{KN} / \mathrm{m}$, the relative error between the natural frequencies with and without the van der Waals force is $39.97 \%$ for mode $(1,1)$. At $F=\sigma_{0 x}=5.5 \times 10^{3} \mathrm{KN} / \mathrm{m}$, 

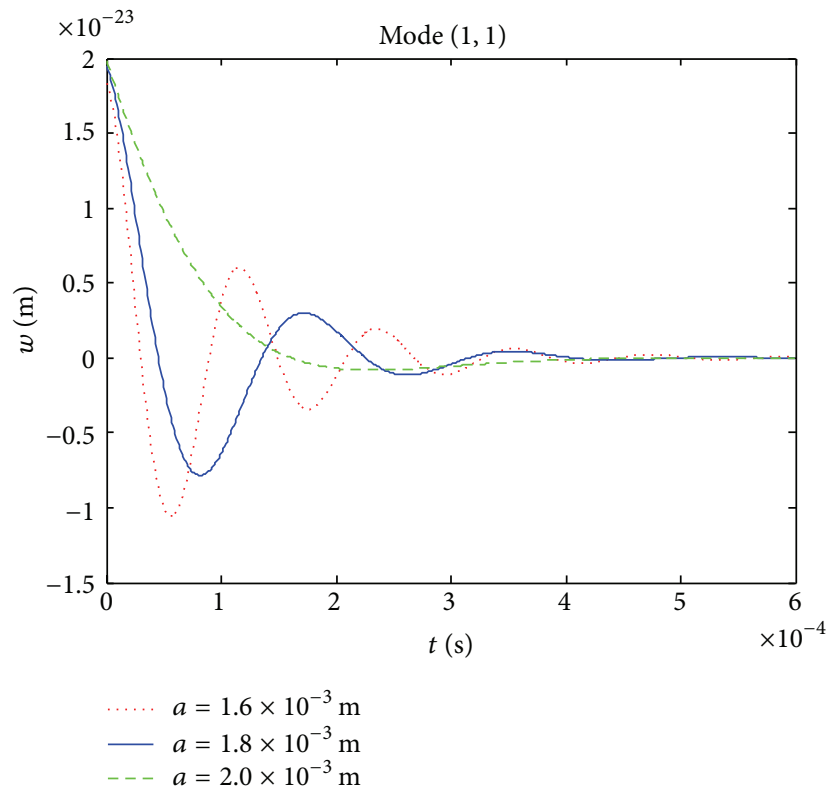

(a) With van der Waals force

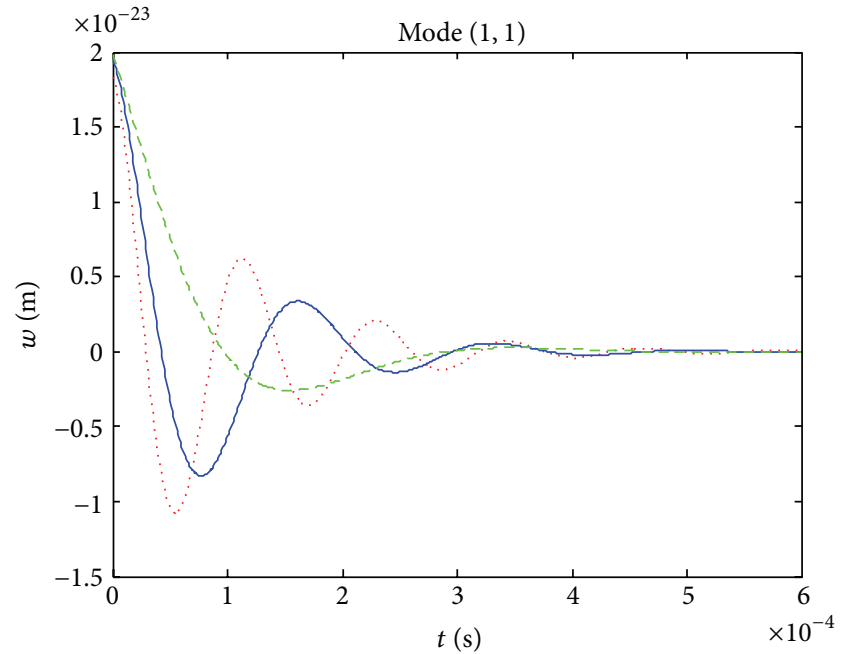

$$
\begin{array}{rl}
\ldots a & a=1.6 \times 10^{-3} \mathrm{~m} \\
-a & =1.8 \times 10^{-3} \mathrm{~m} \\
\ldots a & =2.0 \times 10^{-3} \mathrm{~m}
\end{array}
$$

(b) Without van der Waals force

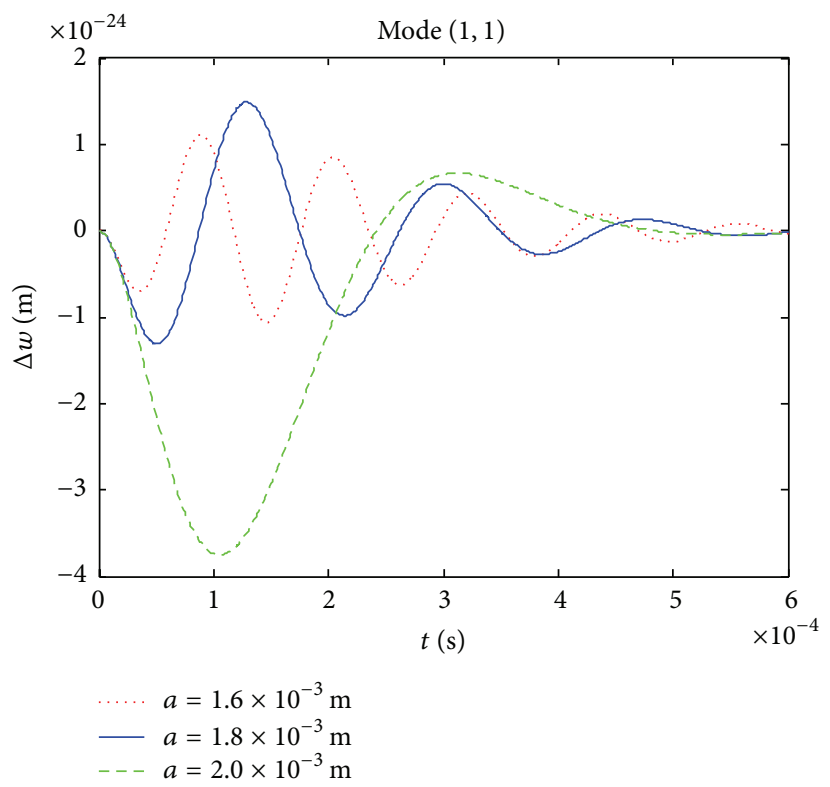

(c) Difference of the vibrating amplitudes

Figure 2: Effects of the van der Waals force on vibrating amplitude of the film center for various $a$.

the relative error between the natural frequencies with and without the van der Waals force is $6.59 \%$ for mode $(1,1)$.

Hence, the effects of the van der Waals force on the natural frequency of the micro resonant pressure sensor should be considered for a small clearance between the film and the base plate, a small initial tension stress of the film, and a low order mode of the vibrations.

The effects of the van der Waals force on the vibrating amplitudes of the film center are investigated for mode $(1,1)$ and various system parameters (see Figures 2-6). They show the following.

(1) Due to the effects of the damping, the free vibration of the film center is periodic vibration with the amplitude decay. As the length $a$ of the micro film grows, the vibrating amplitudes of the film center drop more rapidly with the time. When the van der Waals force is not considered, the vibrating amplitudes of the film center drop more rapidly with time compared to the case when the van der Waals force 


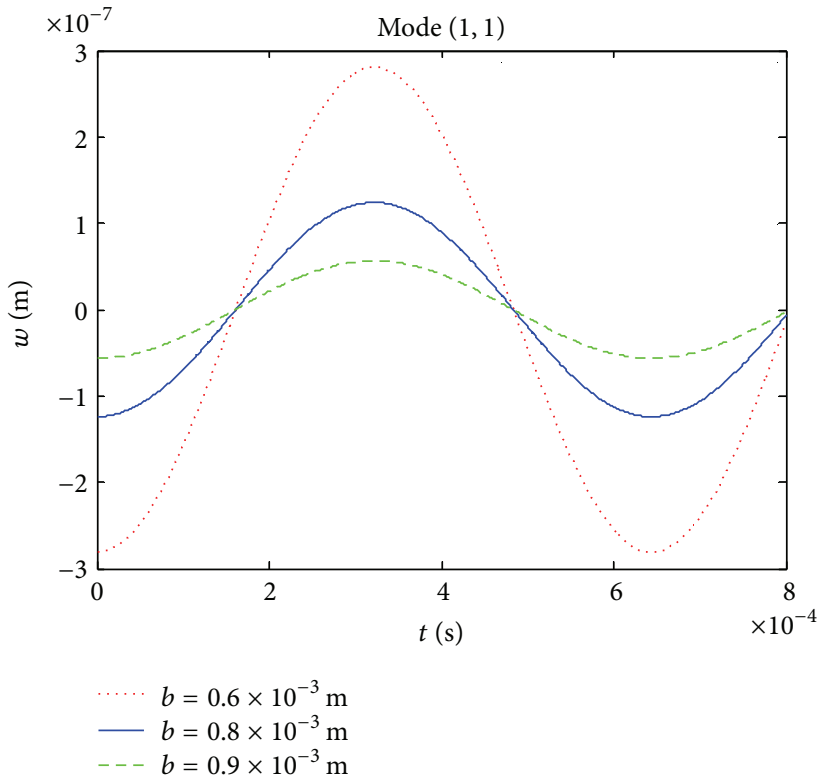

(a) With van der Waals force

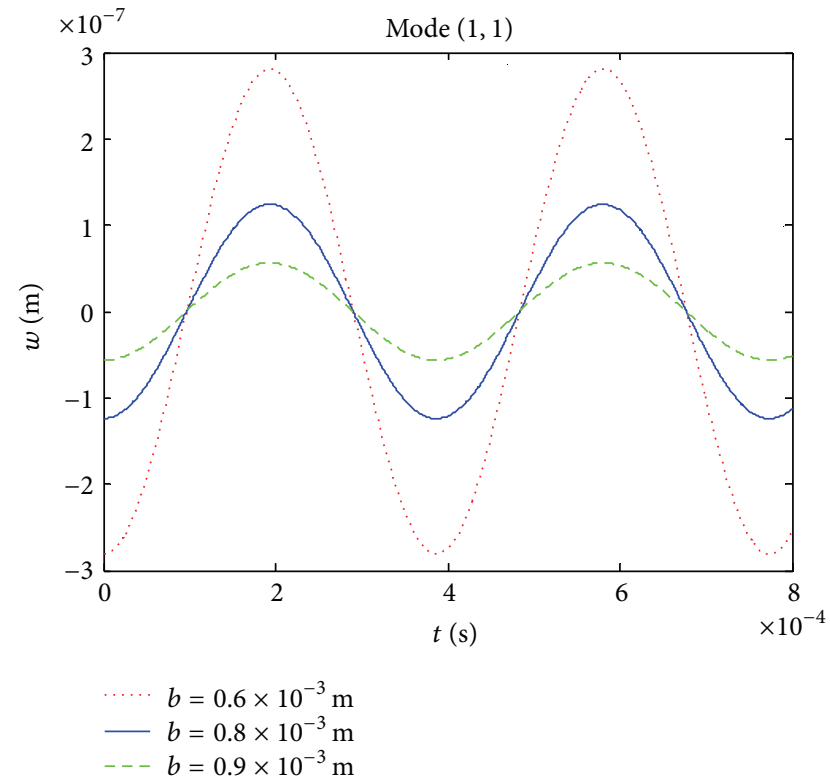

(b) Without van der Waals force

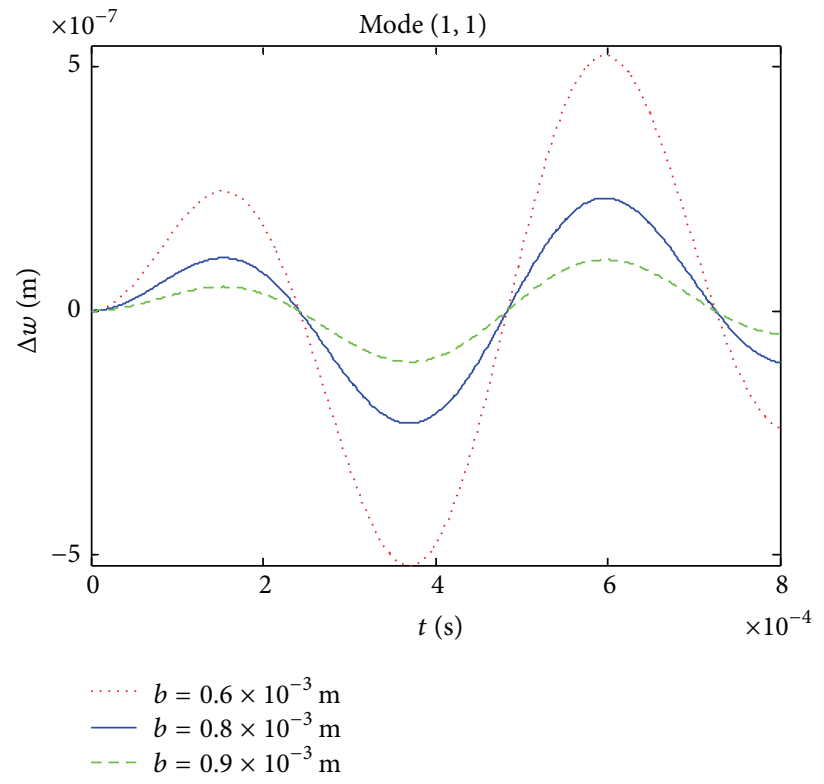

(c) Difference of the vibrating amplitudes

FIGURE 3: Effects of the van der Waals force on vibrating amplitude of the film center for various $b$.

is considered. As the length $a$ of the micro film grows, the effects of the van der Waals force on the vibrating amplitudes of the film center become large.

(2) As the width $b$ of the micro film grows, the vibrating amplitudes of the film center drop. However, the amplitude decay does not occur. When the van der Waals force is not considered, the vibrating amplitudes of the film center are larger than those in the case when the van der Waals force is considered. As the width $b$ of the micro film grows, the effects of the van der Waals force on the vibrating amplitudes of the film center become small.

(3) As the thickness $h$ of the micro film grows, the vibrating amplitudes of the film center drop more slowly with time. When the van der Waals force is not considered, the vibrating amplitudes of the film center drop first more rapidly and then more slowly with time compared to those in the case 


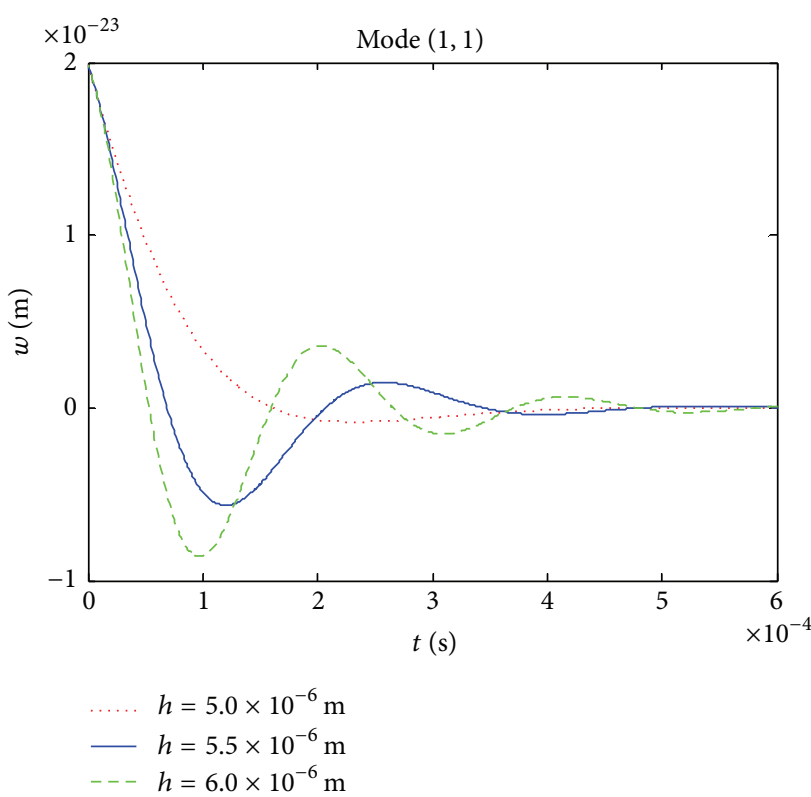

(a) With van der Waals force

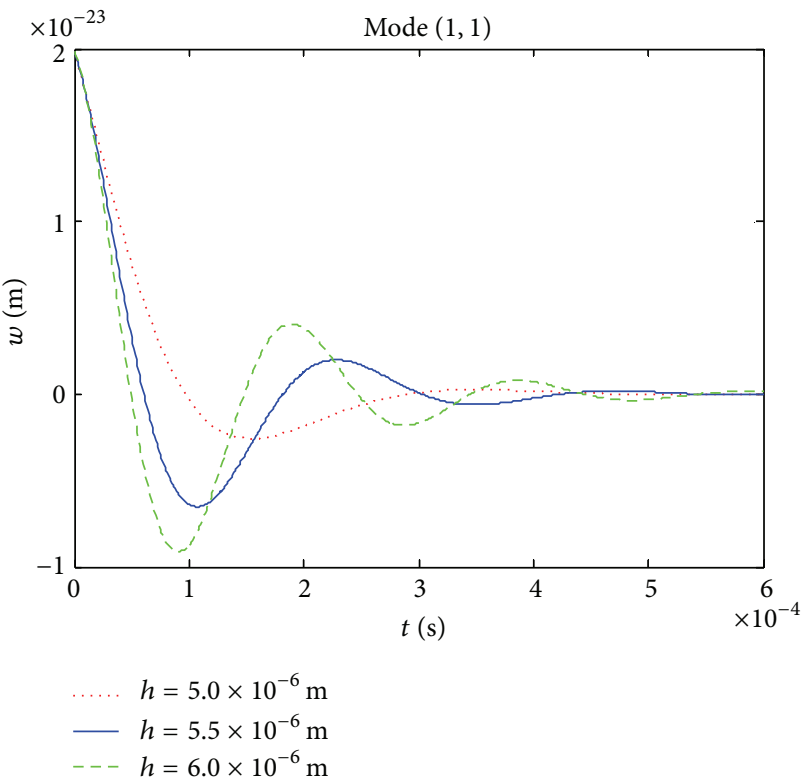

(b) Without van der Waals force

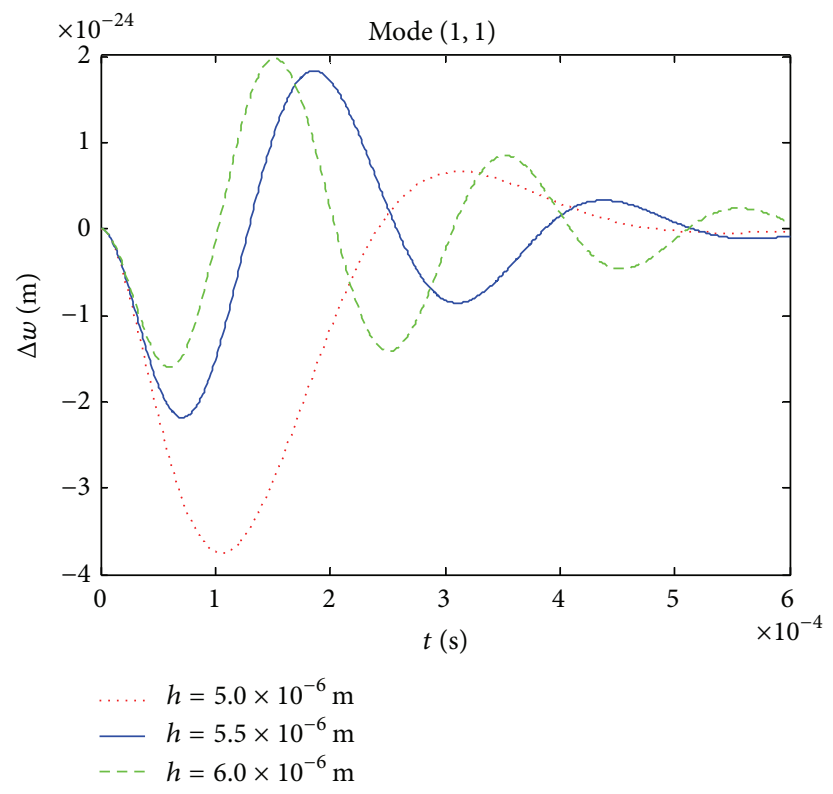

(c) Difference of the vibrating amplitudes

FigURE 4: Effects of the van der Waals force on vibrating amplitude of the film center for various $h$.

when the van der Waals force is considered. As the thickness $h$ of the micro film grows, the effects of the van der Waals force on the vibrating amplitudes of the film center first become small and then become large.

(4) As the initial tension stress of the micro film grows, the vibrating amplitudes of the film center drop more slowly with time. When the van der Waals force is not considered, the vibrating amplitudes of the film center drop first more rapidly and then more slowly with time compared to those in the case when the van der Waals force is considered. As the initial tension stress of the micro film grows, the effects of the van der Waals force on the vibrating amplitudes of the film center first become small and then become large.

(5) As the voltage between the micro film and the back plate grows, the vibrating amplitudes of the film center drop more rapidly with time. When the van der Waals force is 


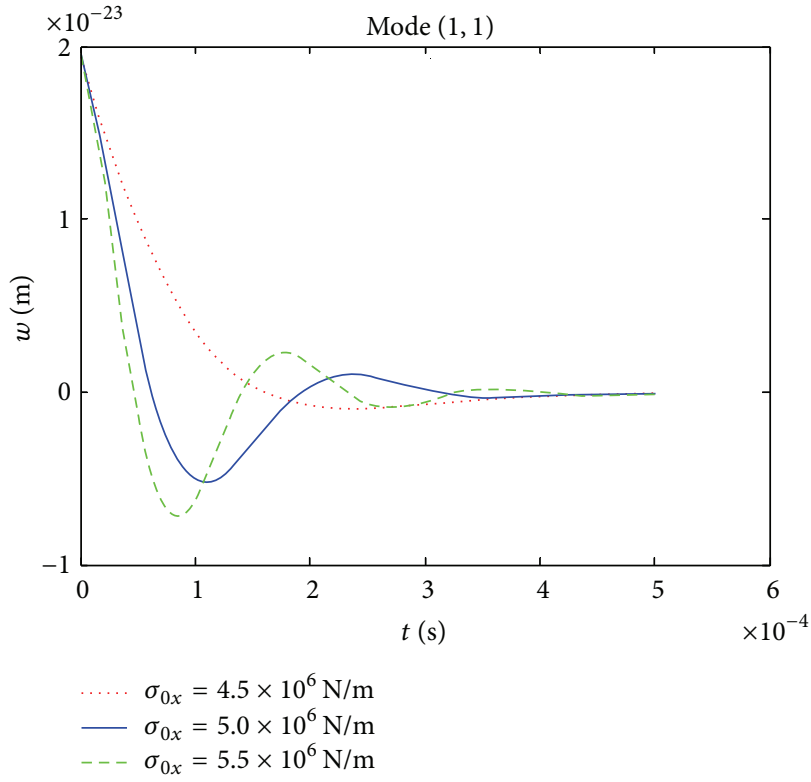

(a) With van der Waals force

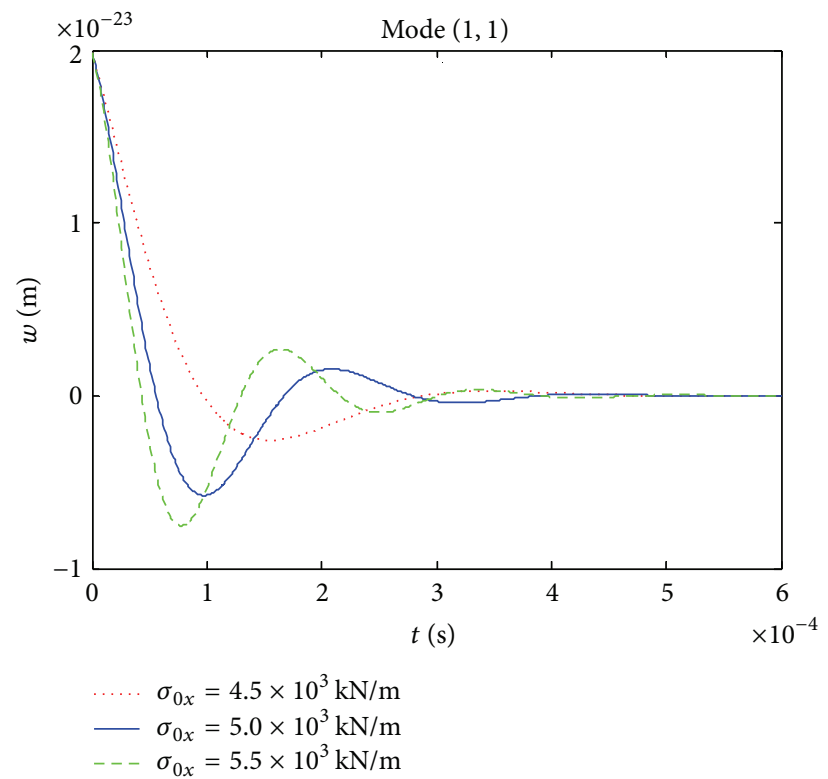

(b) Without van der Waals force

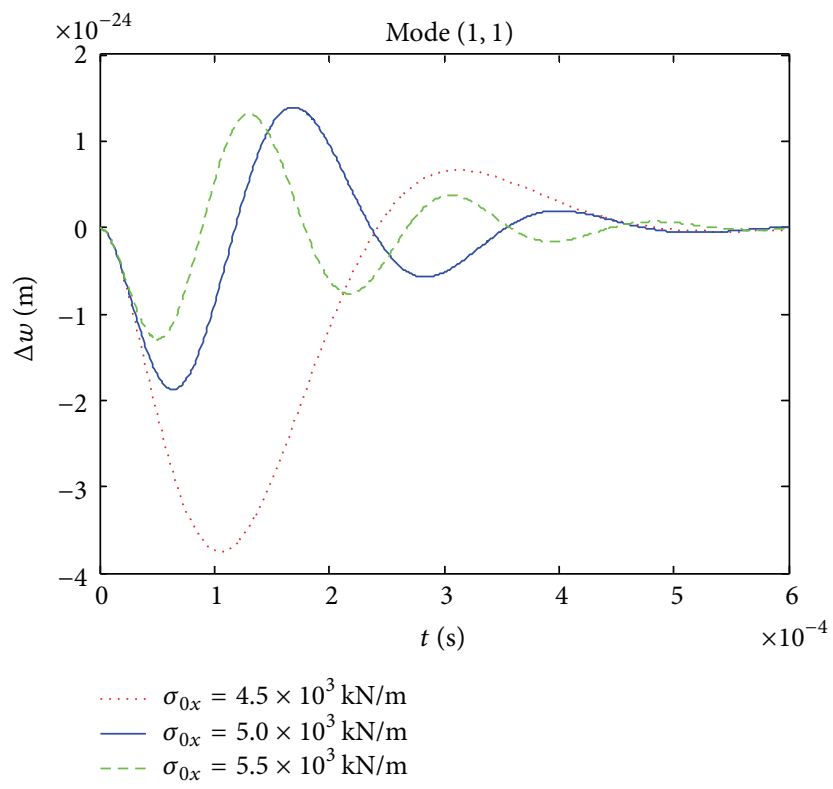

(c) Difference of the vibrating amplitudes

FIGURE 5: Effects of the van der Waals force on vibrating amplitude of the film center for various $\sigma_{0 x}$.

not considered, the vibrating amplitudes of the film center drop more rapidly with time compared to those in the case when the van der Waals force is considered. As the voltage grows, the effects of the van der Waals force on the vibrating amplitudes of the film center become large.

In a word, the effects of the van der Waals force on the vibrating amplitudes of the film become large under some conditions which include a large voltage between the micro film and the back plate, a large film length and thickness, a small film width, and a small initial tension stress of the micro film.

\section{Conclusions}

In this paper, a coupled dynamic model of the micro resonant pressure sensor is proposed and its coupled dynamic equation is given in which the van der Waals force is considered. By the equation, the effects of the van der Waals force on the natural 


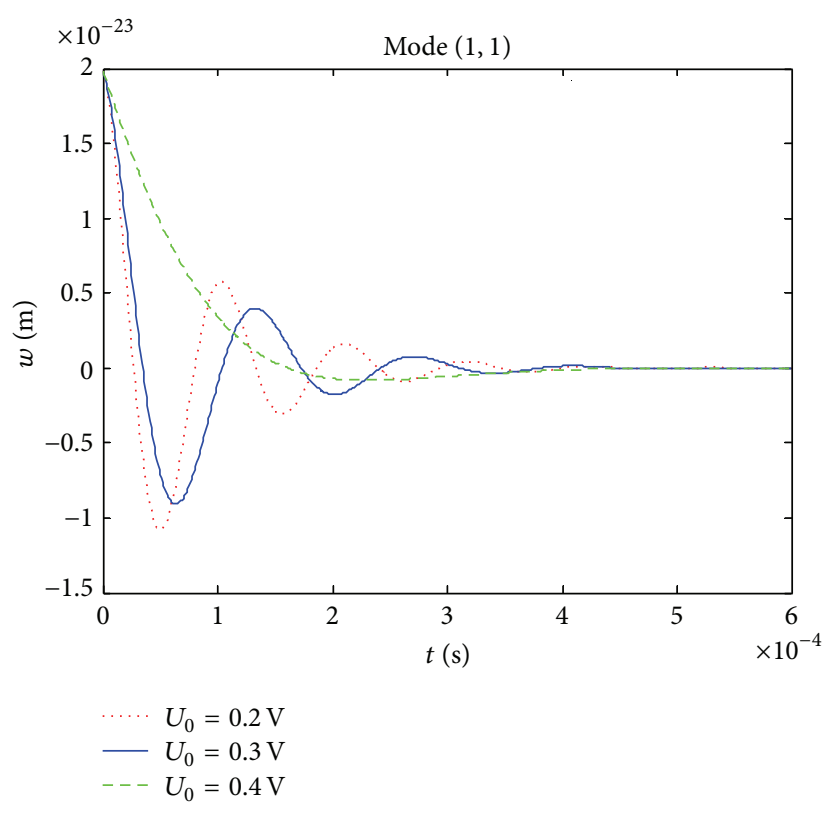

(a) With van der Waals force

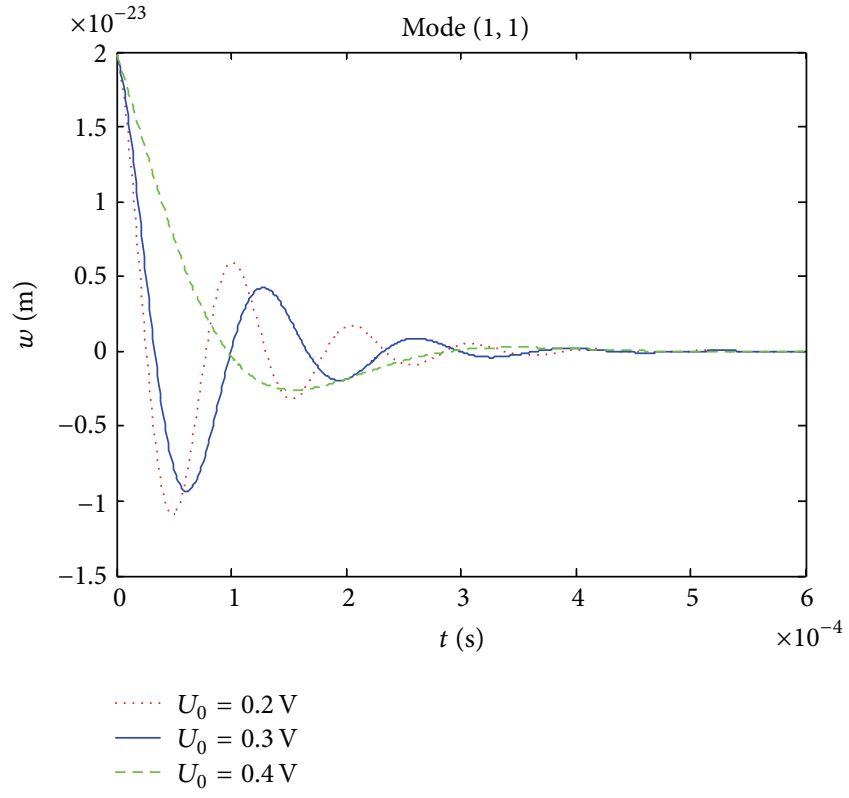

(b) Without van der Waals force

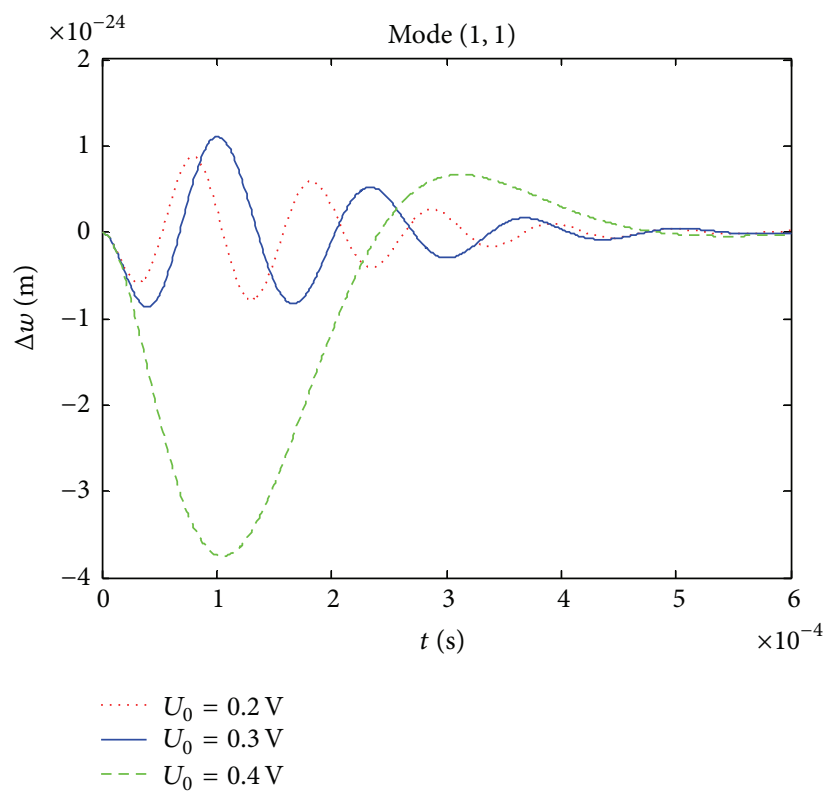

(c) Difference of the vibrating amplitudes

FIGURE 6: Effects of the van der Waals force on vibrating amplitude of the film center for various $U_{0}$.

frequencies and vibration amplitudes of the micro resonant pressure sensor are investigated. Results show the following.

(1) The effects of the van der Waals force on the natural frequency of the micro resonant pressure sensor should be considered for a small clearance between the film and the base plate, a small initial tension stress of the film, and a low order mode of the vibrations.

(2) The effects of the van der Waals force on the vibrating amplitudes of the film should be considered for a large voltage between the micro film and the back plate, a large film length and thickness, a small film width, and a small initial tension stress of the micro film.

\section{Conflict of Interests}

The authors declare that there is no conflict of interests regarding the publication of this paper. 


\section{Acknowledgment}

This project is supported by Key Basic Research Foundation in Hebei Province of China (13961701D).

\section{References}

[1] E. Thielicke and E. Obermeier, "Microactuators and their technologies," Mechatronics, vol. 10, no. 4, pp. 431-455, 2000.

[2] R. Nadal-Guardia, A. M. Brosa, and A. Dehé, "AC transfer function of electrostatic capacitive sensors based on the 1-D equivalent model: application to silicon microphones," Journal of Microelectromechanical Systems, vol. 12, no. 6, pp. 972-978, 2003.

[3] T. S. J. Lammerink, M. Elwenspoek, R. H. Van Ouwerkerk, S. Bouwstra, and J. H. J. Fluitman, "Performance of thermally excited resonators," Sensors and Actuators A, vol. 21, no. 1-3, pp. 352-356, 1990.

[4] E. Donzier, O. Lefort, S. Spirkovitch, and F. Baillieu, "Integrated magnetic field sensor," Sensors and Actuators A: Physical, vol. 26, no. 1-3, pp. 357-361, 1991.

[5] K. E. Petersen, F. Pourahmadi, J. Brown, P. Parsons, M. Skinner, and J. Tudor, "Resonant beam pressure sensor fabricated with silicon fusion bonding," in Proceedings of the International Conference on Solid-State Sensors and Actuators (TRANSDUCERS '91), pp. 664-667, IEEE, San Francisco, Calif, USA, June 1991.

[6] L. Lanotte, V. Iannotti, and M. Müller, "A new amorphous alloy for magnetoelastic waves applications $\mathrm{Fe}_{62.5} \mathrm{Co}_{6} \mathrm{Ni}_{7.5} \mathrm{Zr}_{6} \mathrm{Cu}_{1} \mathrm{Nb}_{2} \mathrm{~B}_{15}$, International Journal of Applied Electromagnetics and Mechanics, vol. 10, no. 3, pp. 215-220, 1999.

[7] C. Yang, C. Guo, and X. Yuan, "Investigation based on nanoelectromechanical system double $\mathrm{Si}_{3} \mathrm{~N}_{4}$ resonant beam pressure sensor," Journal of Nanoscience and Nanotechnology, vol. 11, no. 12, pp. 10854-10858, 2011.

[8] Z. Tang, S. Fan, W. Xing, Z. Guo, and Z. Zhang, "An electrothermally excited dual beams silicon resonant pressure sensor with temperature compensation," Microsystem Technologies, vol. 17, no. 9, pp. 1481-1490, 2011.

[9] Q. Li, S. Fan, Z. Tang, and W. Xing, "Non-linear dynamics of an electrothermally excited resonant pressure sensor," Sensors and Actuators A: Physical, vol. 188, pp. 19-28, 2012.

[10] L. Xu and W. Chang, "Sensitivity for a microresonant beam pressure sensor with docks," Proceedings of the Institution of Mechanical Engineers C: Journal of Mechanical Engineering Science, vol. 227, no. 4, pp. 852-861, 2013.

[11] S. Ren, W. Yuan, D. Qiao, J. Deng, and X. Sun, "Pressure sensor with integrated resonator operating at atmospheric pressure," Sensors, vol. 13, no. 12, pp. 17006-17024, 2013.

[12] Y. Li, D. Chen, J. Wang, and J. Chen, "A new stress isolation method in the packaging of resonant pressure micro sensors," Sensor Letters, vol. 11, no. 2, pp. 264-269, 2013.

[13] A. Koochi, A. Kazemi, F. Khandani, and M. Abadyan, "Influence of surface effects on size-dependent instability of nanoactuators in the presence of quantum vacuum fluctuations," Physica Scripta, vol. 85, no. 3, Article ID 035804, 2012.

[14] A. Koochi, H. Hosseini-Toudeshky, H. R. Ovesy, and M. Abadyan, "Modeling the influence of surface effect on instability of nano-cantilever in presence of Van der Waals force," International Journal of Structural Stability and Dynamics, vol. 13, no. 4, Article ID 250072, 2013.
[15] L. Xu and D. Zhao, "Effects of the molecular forces on the free vibration of electromechanical integrated electrostatic harmonic actuator," Precision Engineering, vol. 37, no. 2, pp. 275285, 2013.

[16] W. J. Song, Study on dynamic response of orthotropic membranes under impact loading [Ph.D. thesis], Chongqing University, Chongqing, China, 2011. 


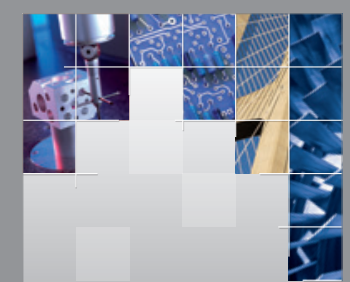

\section{Enfincering}
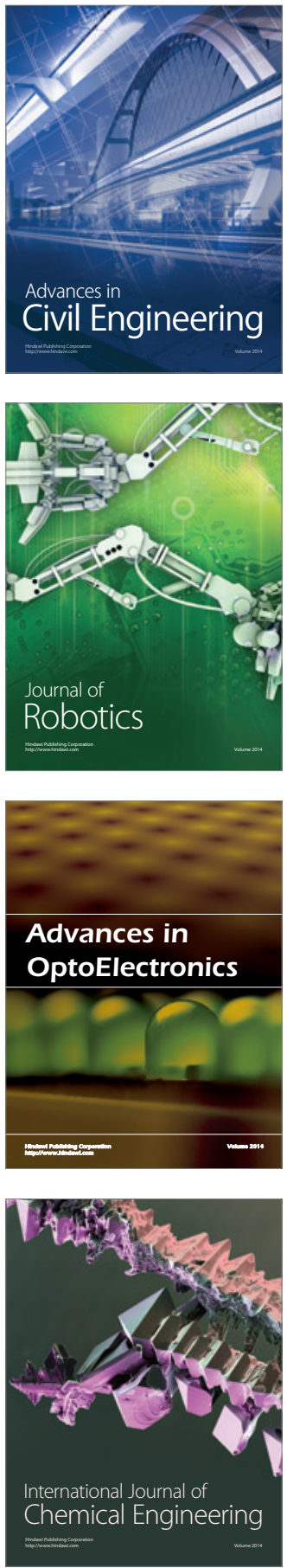

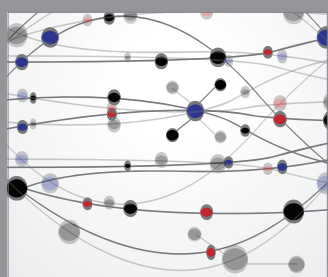

The Scientific World Journal

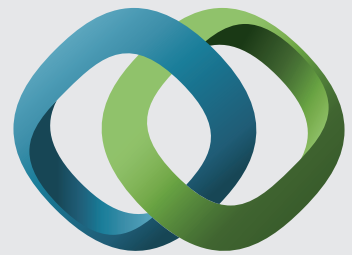

\section{Hindawi}

Submit your manuscripts at

http://www.hindawi.com
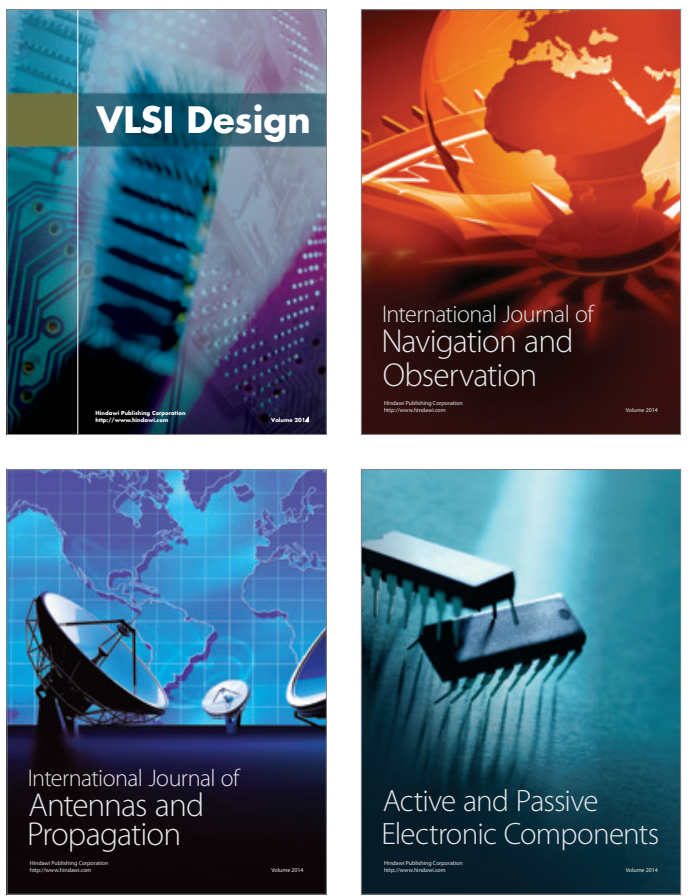
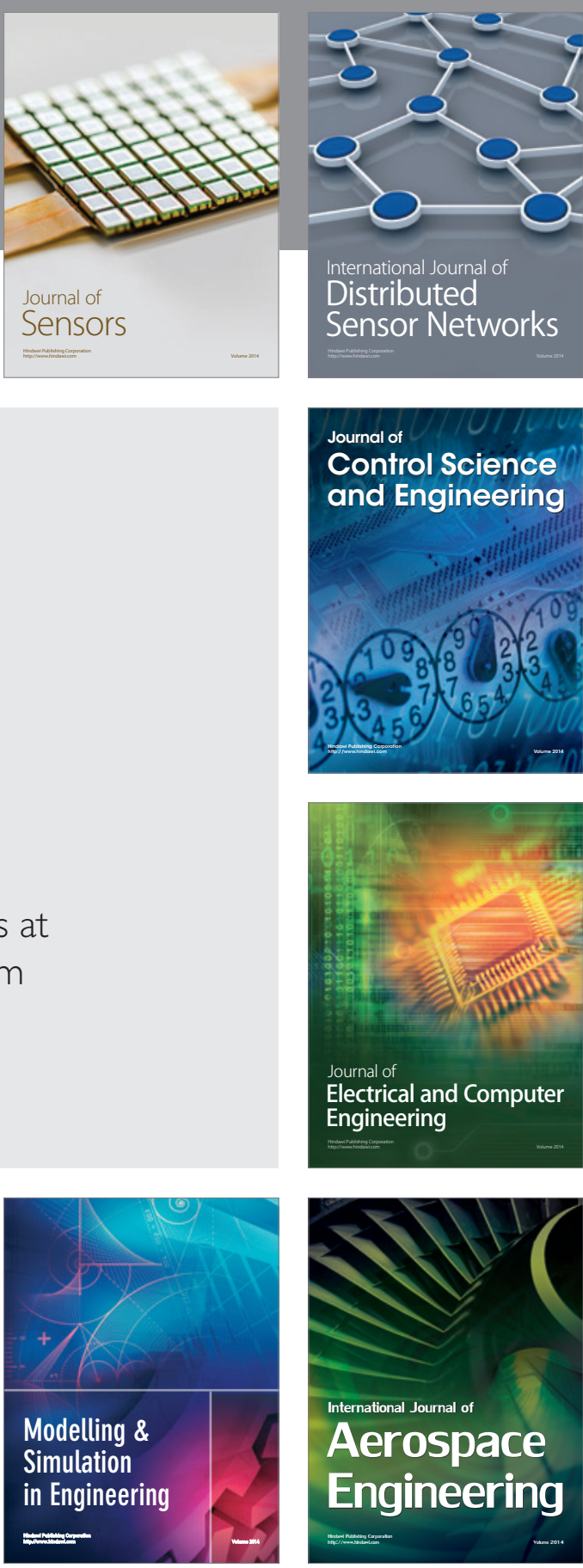

International Journal of

Distributed

Sensor Networks

Journal of

Control Science

and Engineering
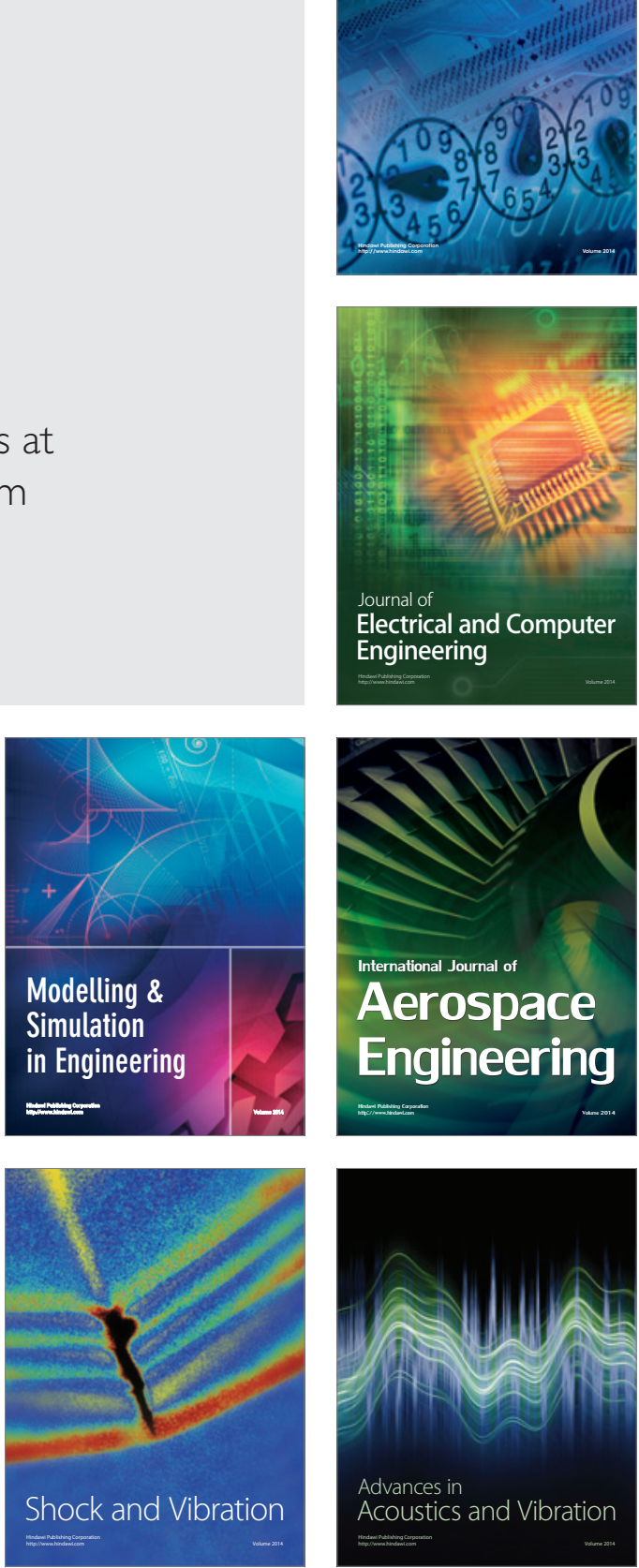